\title{
A Robust OFDM Modem for Underwater Acoustic Communications
}

\author{
Arnaud Bourré, Said Lmai, Christophe Laot and Sébastien Houcke \\ Institut Mines-Telecom ; Telecom Bretagne ; UMR CNRS 6285 Lab-STICC \\ Université Européenne de Bretagne, \\ Technopôle Brest-Iroise - CS 83818 - 29238 Brest Cedex 3, France \\ E-mail: arnaud.bourre@telecom-bretagne.eu
}

\begin{abstract}
The use of orthogonal frequency division multiplexing (OFDM) based underwater acoustic (UWA) modems has raised a growing interest within the scientific community. This is motivated mainly by the relative simple implementation of the OFDM system and its capability to transmit efficiently over long time spread channels. This paper describes the modem developed in the context of COMET project, which aims to produce low cost Autonomous Underwater Vehicles (AUVs) designed to move in an UWA network. This easy configuring software modem is based on a new pulse-shaped OFDM scheme, using the square root raised cosine (RRC) function defined in time domain at both the transmitter and the receiver sides. In addition to its reduced energy consumption, the proposed modem optimizes the spectral efficiency, while neither pilots nor null subcarriers are required with the DPSK modulation use. Flexible and primed to operate with various channel characteristics, it has three different running modes: the standby mode, the locking mode and the decoding mode. To date, the modem is implemented in Matlab language and is real-time running. After the modem presentation in terms of designing modules, we provide supporting results from experiments conducted during sea trials.
\end{abstract}

\section{INTRODUCTION}

The deployment of underwater acoustic (UWA) communication networks in recent years is an open research field [1] gaining more and more interest from both scientific community and industrials. For illustrative examples, we would cite [2]-[4], where raised projects consider several oceanographic applications. While a rapidly deployable UWA network of sensors and Autonomous Underwater Vehicles (AUVs) is the focus in the first project, the second has the purpose to propose a complete solution for the network architecture and the communication protocols required for the tele-operation of underwater robots. Regarding [4], the interest is on a communication infrastructure that offers the flexibility for continuous monitoring of critical infrastructures located by the sea.

To ensure reliable transmissions between nodes, the UWA modem remains a key component. In this paper, we present the modem that has been developed in the context of COMET project, which aims to produce low cost AUVs. An underwater acoustic network allows the AUVs to coordinate navigation and operations. COMET is an officially recognized project of the global economic competitiveness "Pôle Mer Bretagne". COMET is led by RTsys with the partnership of ZTI, Williamson Electronique, ENSTA Bretagne and Télécom

\section{Bretagne.}

The main characteristic of UWA channel is its multipath nature over large duration. In our case, the modem is based on an orthogonal frequency division multiplexing (OFDM) scheme. OFDM is well known for its capability to transmit efficiently over long time spread channels, either with simple one-tap equalization or using more elaborate equalization methods [5]. We had the choice between different techniques like zero-padded (ZP) OFDM [6], cyclic prefix (CP) OFDM or pulse-shaped OFDM. We decide to implement a new pulseshaped OFDM system for several reasons. Firstly, we have no waste of energy since we do not remove the $\mathrm{CP}$ at the receiver. Secondly, it is easy to change the shaping filter to adapt to channel characteristics (increase the used pulse roll-off factor for long impulse response). Lastly, inter-symbol and intercarrier interferences (ISI and ICI respectively) are efficiently reduced, still without any waste of energy [7]. Our modem uses the square root raised cosine (RRC) as pulse-shaping function, defined in time domain for both the transmitter and the receiver.

Another important characteristic of UWA channel is its low time-frequency coherence. Therefore, the channel response estimation requires a high density of pilot symbols [8], [9], which may dramatically decrease the spectral efficiency. To avoid this drawback, we propose to use a differential phaseshift keying (DPSK) modulation, where the data are carried out on each subcarrier by the phase difference between two PSK symbols belonging to the successive OFDM symbols. With such a modulation, we do not need any channel estimation [10] and save precious spectral efficiency. Besides, convolutional coding and bit interleaving are employed mainly to protect each OFDM symbol against frequency selective fading.

A third essential characteristic of UWA channel is that Doppler shift is non-uniform across all subcarriers. Therefore, compensating by a simple phase correction in time domain, like for narrow-band signals, is not convenient. It is of common use to take on resampling for reconstructing a Doppler free signal [11], [12]. However, in our modem, the received signal is projected on expanded or compressed versions of analysis functions for which analytical expressions are available. In order to prevent a loss in spectral efficiency, Doppler factor is estimated by exploiting channel amplitude spectra correlation between two successive symbols. This 
means that there is no need to reserve null subcarriers for Doppler compensation [6]. The knowledge of Doppler factor enables us to determine, not only the current symbol period but also the beginning of the following OFDM symbol. Thus, time and frequency shifts are jointly corrected.

The proposed modem is energy efficient. It has actually three different running modes: standby mode, locking mode and decoding mode. During the standby mode, the receiving filter output power is measured on each symbol period until a certain threshold is reached. For this mode, computational cost is low in order to save batteries lifetime. During the locking mode, classical synchronization operations are performed using a unique OFDM symbol as preamble: identification of the start of the first OFDM symbol and Doppler factor estimation. This symbol determines the initial phases for DPSK modulation. During decoding mode, for each OFDM symbol, the Doppler factor is updated and the phase differences are estimated. An angular correction is made to reduce the phase jitter of DPSK symbols.

The remainder of the paper is organized as follows. In Section II, we present the proposed UWA modem is terms of designing modules. We introduce the experiment setup description in Section III. Section IV presents the synthesis of the experimental results. Finally, conclusions (and future work) are summarized in Section V.

\section{MODEM DESIGN}

\section{A. Modulator}

In an OFDM system, the sub-carrier spacing $B$ is obtained based on the "useful" OFDM symbol duration $T_{o}$ (without CP) i.e. $B=1 / T_{o}$. Let $T$ be the nominal duration of the OFDM symbol defined as: $T=T_{o}+T_{g}$, where $T_{g}$ denotes the CP length (or the equivalent guard interval in case of $\mathrm{ZP}$ OFDM). With respect to the ZP-OFDM scheme, the symbol pulse duration at the transmitter is: $T_{o}$, while the analysis window duration at the reception side is: $T>T_{o}$. However, regarding the CP-OFDM scheme, the symbol pulse duration is: $T$, while the analysis window duration is: $T_{o}<T$. For our modem, the used OFDM system is built using the pulseshaping function $g(t)$, the RRC, implemented at both sides: transmission and reception modules. Furthermore, the symbol pulse duration is: $T$ (CP included) and the analysis window duration is: $\frac{1+\alpha}{B}=T$, where $\alpha$ is the roll-off factor of $g(t)$ and $\alpha \in[0,1]$. In other words, the synthesis window at the transmitter and the analysis window at the receiver have the same size. It should be noted that the roll-off period length corresponds exactly to $T_{g}$ i.e. $T_{g}=\alpha T_{o}$. Hence, we have: $B T=1+\alpha$.

$g(t)$ is defined by:

$$
g(t)=\sqrt{B} \cdot \begin{cases}0, & t<0 \\ \sin \left(\frac{\pi}{2} \frac{B}{\alpha} t\right), & 0 \leq t<\frac{\alpha}{B} \\ 1, & \frac{\alpha}{B} \leq t<\frac{1}{B} \\ \sin \left[\frac{\pi}{2} \frac{B}{\alpha}(T-t)\right], & \frac{1}{B} \leq t<T \\ 0, & t \geq T\end{cases}
$$

The orthogonal basis functions used for the synthesis of the signal at the transmitter, are given by:

$$
\begin{aligned}
& g_{n}(t)=g(t) \exp \left(j 2 \pi f_{n} t\right), \quad t \in[0, T] \\
& n=0,1, \ldots, N-1
\end{aligned}
$$

where $f_{n}$ is the frequency of the $n^{t h}$ subcarrier.

The continuous-time complex envelope of the transmitted signal may be written as:

$$
s(t)=\sum_{k=0}^{+\infty} \sum_{n=0}^{N-1} d_{k, n} g_{n}(t-k T) .
$$

where $d_{k, n}$ is the DPSK information symbol transmitted on the $n^{t h}$ subcarrier of the $k^{t h}$ OFDM symbol.

Therewith, we employ a convolutional code and a bit interleaving scheme to obtain the robustness needed for each OFDM symbol against frequency selective fading. The transmission takes advantage of the characteristics of DPSK modulation. As no channel estimation or tracking is required, there is a gain in spectral efficiency.

One should notice here that, for a given average signal to noise ratio $(S N R)$, the use of the pulse-shaping reduces the energy, in time domain, on the edges of the designed OFDM symbol. It allows to place the ISI in signal regions of low SNR. Once implemented at the receiver side, the pulse-shaping mitigates even more the energy of ISI-affected samples [7]. Furthermore, the used pulse frequency spread is: $\frac{B}{4 \sqrt{\alpha}}$. Thus, choosing high values for the parameter $\alpha$ makes it possible to reduce the ICI due to the channel frequency spread.

In Fig. 1, the transmitter and receiver block diagram depicts the basic modules that compose the modem.

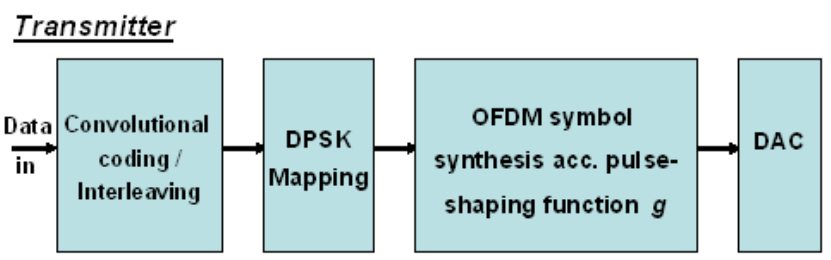

\section{Receiver}

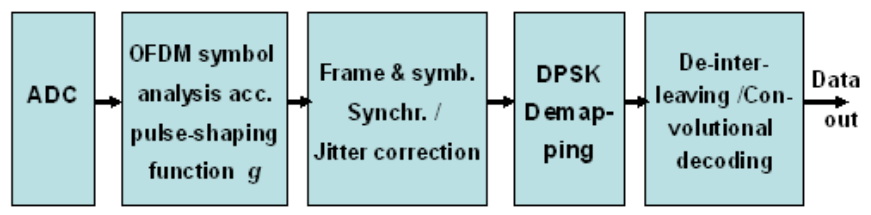

Fig. 1. Transmitter and receiver block diagram.

\section{B. Demodulator}

1) Projection-based analysis: Unlike the conventional CPOFDM scheme, we do not remove the $\mathrm{CP}$ at the receiver side. Actually, we first perform the received signal projection onto a set of expanded or compressed versions of basis functions for 
which analytical expressions are available, instead of seeking to reconstruct a Doppler free signal. Let $D$ be the Doppler factor defined as the ratio between the received frequency and the transmitted frequency and let $t$ be the starting time of the analysis window. The outcome of the projection operation is described by:

$$
y_{n}(D, t)=\sqrt{D} \int_{-\infty}^{+\infty} y(\tau) g_{n}^{*}(D(\tau-t)) d \tau .
$$

where $y(\tau)$ is the signal at the input of the receiver and $g^{*}(\tau)$ is the complex conjugate of $g(\tau)$.

2) Frame and symbol sychronization: Regarding the frame synchronization, we exploit the channel coherence in frequency domain. To this end, we use the first OFDM symbol that is known to the receiver. We assume that the Doppler variations are very small from the beginning to the end of each OFDM symbol. Based on a criterion that is employed to properly use the channel coherence, the Doppler factor $D$ and the starting time $t$ of the OFDM symbol, are optimized jointly. Therefore, the initial Doppler factor $D_{o}$ and the starting time of the transmitted frame $t_{o}$ correspond to the values that maximize the estimated channel coherence.

Now, using the nominal duration of the OFDM symbol $T$, the starting time $t_{k}$ and the Doppler factor $D_{k}$ of the previous OFDM symbol, we deduce the starting time $t_{k+1}$ of the new OFDM symbol. However, the new Doppler factor $D_{k+1}$ is determined exploiting the channel coherence in time domain between two consecutive OFDM symbols.

3) Jitter correction: In order to reduce the phase jitter of DPSK symbols resulting from the time variant fading environment, an angular correction is applied over the entire OFDM symbol. We consider the parameter $z_{k, n}$ whose magnitude might be likened to a power, given by:

$$
z_{k, n}=y_{k, n} y_{k-1, n}^{*}, \quad n=0,1, \ldots, N-1
$$

where $y_{k, n}$ is the signal corresponding to the $n^{\text {th }}$ element of the $k^{t h}$ OFDM symbol after the analysis and synchronization operations. Let $\phi_{k}$ be the value of the phase correction to be made. For each OFDM symbol, it is assumed to be the same over the DPSK symbols on all subcarriers. Thereby, we have:

$$
z_{k, n}^{\prime}=z_{k, n} e^{-i \phi_{k}}, \quad n=0,1, \ldots, N-1
$$

where $z_{k, n}^{\prime}$ holds the effective inputs for soft decoding after phase correction. In case of $M$-order modulation, the applied angular correction to compensate the phase jitter involves the weighting terms $\left|z_{k, n}\right|$ and is expressed by:

$$
\phi_{k}=\frac{1}{M} \operatorname{Arg}\left(\sum_{n=0}^{N-1}\left|z_{k, n}\right| e^{j M \arg z_{k, n}}\right) \text {. }
$$

4) Standby: It is the default mode when all executed processing tasks should be low power consumption in order to optimize batteries lifetime. In fact, during this mode, the receiving filter output power is measured over a time hopping window having one OFDM symbol period length. Once a predefined threshold is reached, the modem switches into the locking mode.

5) Locking: The locking phase is crucial for the proper receiver operation in doubly-selective channel. As noticed earlier, the channel coherence estimation is based on the first OFDM symbol detection. This OFDM symbol, known to the receiver, carries the initial phases drawn randomly. Hence, we come up with the channel coherence bandwidth $B_{c}$. It should be greater than the subcarrier spacing $B$ to switch to the decoding mode. Actually, this condition is implemented using the computed correlation coefficient between channel frequency responses at $B$-spaced frequencies, which must exceed a predefined threshold.

\section{DESCRIPTION OF THE EXPERIMENTAL SETUP}

The modem has been evaluated in Brest harbor shallow water, France during sea trials conducted on February $15^{\text {th }}, 2013$. The transmissions were realized between two docks over a $800 \mathrm{~m}$ distance. At the transmitter side, two omnidirectional transducers were successively used: ITC-1001 $(14.8 \mathrm{kHz}-$ $20.2 \mathrm{kHz}$ bandwidth) and ITC-1032 (28.3 kHz - $40.1 \mathrm{kHz}$ bandwidth). The signals were synthetized by a laptop computer and amplified by a Krohn-Hite 7500 wideband amplifier. At the receiver side, four broadband omnidirectional B\&K8106 hydrophones were vertically deployed with a hydrophone spacing of $1 \mathrm{~m}$. The received signals were conditioned and amplified by a B\&K-2690 conditioning amplifier and digitized using an NI-6356 acquisition card.

A view of the area where the experiments were conducted is shown in Fig. 2.

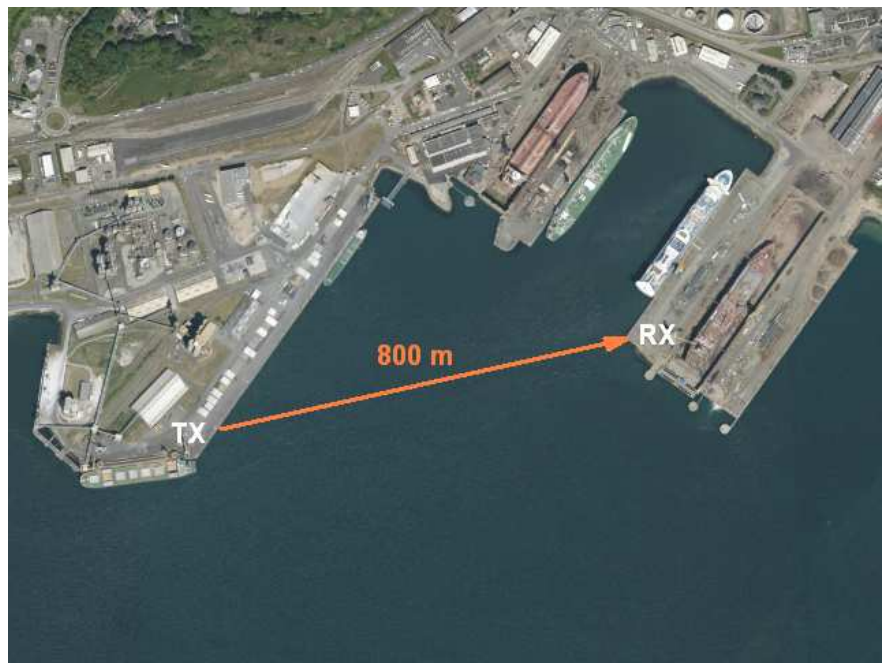

Fig. 2. Sea trials area. 
After the rate $R$ convolutional coding, a pseudo-random scheme, sizing one OFDM symbol length, is used for the bit interleaving. The roll-off factor was fixed at $1 / 3$ and the other parameters are:

- frequency band: $15 \mathrm{kHz}-20 \mathrm{kHz}$ and $30 \mathrm{kHz}-40 \mathrm{kHz}$

- transmission power: $160 \mathrm{~dB}$ (rel. to $1 \mu \mathrm{Pa}$ at $1 \mathrm{~m}$ ) and $150 \mathrm{~dB}$ (rel. to $1 \mu \mathrm{Pa}$ at $1 \mathrm{~m}$ )

- OFDM symbol duration: $40 \mathrm{~ms}$ and $80 \mathrm{~ms}$

- modulation scheme: differential binary PSK (D-BPSK) and differential quadrature (D-QPSK)

- coding rate: $1 / 2$ and $2 / 3$ obtained by puncturing

Sea trials transmissions concern frames of $4.8 \mathrm{~s}$ corresponding to message lengths ranging from 41400 bits to 238200 bits. Data received on each hydrophone are independently processed without multi-channel combining.

\section{EXPERIMENTAL RESULTS}

It is noteworthy that the harbor was experiencing an intense ships activity that introduce considerable variations in the channel. Accordingly, the subcarriers orthogonality would be destroyed and result in ICI.

Results synthesis from sea trials are summarized in Tables I, II, III and IV. In each table, the first column indicates the number of subcarriers $N$, the used modulation (D-BPSK or D-QPSK) and the coding rate $(R)$. The second field of the table the achieved user throughput in bits/s while the four remaining columns show the displayed bit error rate $\left(B E R_{i}\right)$ where $B E R_{i}$ denotes the $B E R$ of the hydrophone $i$. The provided user throughput does not take into account the bits corresponding to the initial OFDM symbol and tail bits. It is given by:

$$
D_{b}=\frac{N B}{1+\alpha} R \log _{2}(M)
$$

The first remark to make is that going from the $15 \mathrm{kHz}$ $20 \mathrm{kHz}$ band to $30 \mathrm{kHz}-40 \mathrm{kHz}$ band, which is twice larger, the produced power spectral level is divided by two in case of same transmission power. Moreover, the distance-dependent absorption in the second band is much more important than that in the first band. However, the encountered noise level in the first band is greater. Ultimately, in terms of $S N R$, there is around $3 \mathrm{~dB}$ difference obtained after calculation to the benefit of the $15 \mathrm{kHz}-20 \mathrm{kHz}$ band. This should explain the degradation in terms of BER between Tables I and II on one side and tables III and IV on the other side respectively.

Looking closely at these results, we realize that, as intended, the general trend is often the higher the throughput the more significant the $B E R$. Nevertheless, zero error is achieved over almost all hydrophones for certain sets of parameters.

Another observation to make when comparing Tables I and III on the one hand with tables II and IV on the other hand respectively, is that decreasing the transmission power by $10 \mathrm{~dB}$ generates a loss in terms of $B E R$.

Let's now take for instance the Table I. In the down part (300 D-PSK), compared to the upper part (150 D-PSK), the
TABLE I

SEA TRIALS RESULTS IN $15 \mathrm{KHZ} \mathrm{-} 20 \mathrm{KHZ}$ BAND, TRANSMISSION POWER: 170 DB (REL. TO $1 \mu$ PA AT $1 \mathrm{M}$ )

\begin{tabular}{|c|c||c|c|c|c|}
\hline OFDM symbol & bit/s & $B E R_{1}$ & $B E R_{2}$ & $B E R_{3}$ & $B E R_{4}$ \\
\hline \hline 150 D-BPSK 1/2 & 1875 & 0 & 0 & 0 & 0.0003 \\
150 D-BPSK 2/3 & 2500 & 0 & 0.002 & 0 & 0.0009 \\
150 D-QPSK 1/2 & 3750 & 0 & 0.0003 & 0 & 0.004 \\
150 D-QPSK 2/3 & 5000 & 0.0002 & 0.02 & 0.0009 & 0.09 \\
\hline 300 D-BPSK 1/2 & 1875 & 0 & 0 & 0 & 0 \\
300 D-BPSK 2/3 & 2500 & 0 & 0 & 0 & 0.00003 \\
300 D-QPSK 1/2 & 3750 & 0 & 0 & 0 & 0 \\
300 D-QPSK 2/3 & 5000 & 0 & 0.0003 & 0 & 0.006 \\
\hline
\end{tabular}

TABLE II

SEA TRIALS RESULTS IN $15 \mathrm{KHZ}$ - $20 \mathrm{KHZ}$ BAND, TRANSMISSION POWER: $160 \mathrm{DB}$ (REL. TO $1 \mu$ PA AT $1 \mathrm{M})$

\begin{tabular}{|c|c||c|c|c|c|}
\hline OFDM symbol & bit/s & $B E R_{1}$ & $B E R_{2}$ & $B E R_{3}$ & $B E R_{4}$ \\
\hline \hline 150 D-BPSK 1/2 & 1875 & 0 & 0.0005 & 0 & 0.0002 \\
150 D-BPSK 2/3 & 2500 & 0.006 & 0.02 & 0 & 0.008 \\
150 D-QPSK 1/2 & 3750 & 0.002 & 0.01 & 0 & 0.007 \\
150 D-QPSK 2/3 & 5000 & 0.07 & 0.2 & 0.002 & 0.1 \\
\hline 300 D-BPSK 1/2 & 1875 & 0 & 0 & 0 & 0 \\
300 D-BPSK 2/3 & 2500 & 0.0009 & 0.002 & 0 & 0.00005 \\
300 D-QPSK 1/2 & 3750 & 0.0002 & 0 & 0 & 0 \\
300 D-QPSK 2/3 & 5000 & 0.006 & 0.02 & 0 & 0.01 \\
\hline
\end{tabular}

TABLE III

SEA TRIALS RESULTS IN $30 \mathrm{KHZ}-40 \mathrm{KHZ}$ BAND, TRANSMISSION POWER: 170 DB (REL. TO $1 \mu$ PA AT $1 \mathrm{M}$ )

\begin{tabular}{|c|c||c|c|c|c|}
\hline OFDM symbol & bit/s & $B E R_{1}$ & $B E R_{2}$ & $B E R_{3}$ & $B E R_{4}$ \\
\hline \hline 300 D-BPSK 1/2 & 3750 & 0 & 0.00007 & 0 & 0 \\
300 D-BPSK 2/3 & 5000 & 0.0002 & 0.001 & 0.0001 & 0 \\
300 D-QPSK 1/2 & 7500 & 0.0003 & 0.005 & 0.00004 & 0 \\
300 D-QPSK 2/3 & 10000 & 0.02 & 0.06 & 0.003 & 0.002 \\
\hline 600 D-BPSK 1/2 & 3750 & 0 & 0 & 0 & 0 \\
600 D-BPSK 2/3 & 5000 & 0 & 0.0002 & 0 & 0 \\
600 D-QPSK 1/2 & 7500 & 0 & 0.0004 & 0 & 0 \\
600 D-QPSK 2/3 & 10000 & 0.0004 & 0.008 & 0.00001 & 0 \\
\hline
\end{tabular}

reached throughput is the same but the $B E R$ is at least one order better. This is because the roll-off period of $20 \mathrm{~ms}$ corresponding to the second line is twice larger. Hence, the channel time delay spread is greater than $10 \mathrm{~ms}$, owing to the ineluctable acoustic reverberation phenomenon.

Furthermore, over the $15 \mathrm{kHz}-20 \mathrm{kHz}$ band, if we make the comparaison between the performance of D-BPSK and D-QPSK modulations with different coding rates, we see that: almost on each of the four hydrophones, the D-QPSK modulation in case of $1 / 2$ coding rate achieves better $B E R$ and higher throughput than the D-BPSK modulation in case of $2 / 3$ coding rate. This is may be due to the suboptimality of the second coding scheme where rate $2 / 3$ is constructed by puncturing. 
TABLE IV

SEA TRIALS RESULTS IN $30 \mathrm{KHZ}-40 \mathrm{KHZ}$ BAND, TRANSMISSION POWER: $160 \mathrm{DB}$ (REL. TO $1 \mu \mathrm{PA}$ AT $1 \mathrm{M}$ )

\begin{tabular}{|c|c||c|c|c|c|}
\hline OFDM symbol & bit/s & $B E R_{1}$ & $B E R_{2}$ & $B E R_{3}$ & $B E R_{4}$ \\
\hline \hline 300 D-BPSK & 3750 & 0.006 & 0.2 & 0 & 0.00003 \\
300 D-BPSK & 5000 & 0.01 & 0.02 & 0.004 & 0.005 \\
300 D-QPSK & 7500 & 0.02 & 0.02 & 0.004 & 0.01 \\
300 D-QPSK & 10000 & 0.09 & 0.09 & 0.03 & 0.08 \\
\hline 600 D-BPSK & 3750 & 0.002 & 0.005 & 0 & 0 \\
600 D-BPSK & 5000 & 0.008 & 0.02 & 0.004 & 0.0004 \\
600 D-QPSK & 7500 & 0.02 & 0.03 & 0.007 & 0.002 \\
600 D-QPSK & 10000 & 0.07 & 0.1 & 0.03 & 0.04 \\
\hline
\end{tabular}

\section{CONCLUSIONS}

In this paper, we present the UWA modem developed in the context of COMET project. With respect to this softwaredefined modem, both the transmitter and the receiver implement a new pulse-shaped OFDM method using the RRC function. In addition, the other main modules use a convolutional coding, a random bit-interleaving scheme, a differential PSK modulation and adapted frame and symbol synchronization techniques. The modem operates in three modes: the standby mode, the locking mode and the decoding mode. During sea trials and under multiple parameter sets, the developed modem has been experimentally tested. Although four hydrophones were on the reception side, each hydrophone recordings are processed separately. Besides the succesful detection of all transmitted frames, the achieved performance in terms $B E R$ are very encouraging and confirm the robustness of the proposed modem.

Nevertheless, further consideration of alternatives to improve the receiver performance would be explored. For instance, a maximal ratio combining system for diversity reception is an attractive orientation. Moreover, we look to develop and enhance the network capability of the modem, especially with regard to the media access scheme efficiency and the network throughput maximization.

\section{ACKNOWLEDGMENT}

This work has been supported in part by the "Fonds unique interministériel" (FUI) France within the COMET project.
The authors would particularly like to thank the Brest harbor authorities for having facilitated access to the sea trials area and for all of their efforts to ensure that the experiments are carried out under the best conditions.

\section{REFERENCES}

[1] J. Heidemann, M. Stojanovic, and M. Zorzi, "Underwater sensor networks: Applications, advances, and challenges", Phil. Trans. R. Soc. A, pp. 158-175, Jan. 2012.

[2] J. Kalwa, "The racun-project: Robust acoustic communications in underwater networks - an overview", in Proc. MTS/IEEE OCEANS Conf., Santander, Spain, June 2011.

[3] R. Masiero, P. Casari, and M. Zorzi, "The nautilus project: Physical parameters, architectures and network scenarios", in Proc. MTS/IEEE OCEANS Conf., Kona, Hawaii, USA, Sept. 2011.

[4] A. Caiti, V. Calabro, L. Fusini, A. Munafo, K. Grythe, J.M. Hovem, A. Lie, and T.A. Reinen, "Underwater acoustic network performance: Results from the uan11 sea trial", in Proc. MTS/IEEE OCEANS Conf., Virginia Beach, VA, USA, Oct. 2012.

[5] K. Yeo, K. Pelekanakis, and M. Chitre, "Time-domain equalization for underwater acoustic ofdm systems with insufficient cyclic prefix", in Proc. MTS/IEEE OCEANS Conf., Santander, Spain, June 2011.

[6] Baosheng Li, Shengli Zhou, M. Stojanovic, L. Freitag, and P. Willett, "Multicarrier communication over underwater acoustic channels with nonuniform doppler shifts", IEEE J. Oceanic Eng., vol. 33, no. 2, pp. 198 -209, Apr. 2008.

[7] S. Imai, A. Bourre, C. Laot, and S. Houcke, "Advantages of pulseshaping applied to ofdm systems over underwater acoustic channels", in Proc. MTS/IEEE OCEANS Conf., Virginia Beach, VA, USA, Oct. 2012.

[8] C.R. Berger, Shengli Zhou, J.C. Preisig, and P. Willett, "Sparse channel estimation for multicarrier underwater acoustic communication: From subspace methods to compressed sensing", IEEE Trans. Signal Process., vol. 58, no. 3, pp. 1708-1721, Mar. 2010.

[9] C.R. Berger, J. Gomes, and J.M.F. Moura, "Study of pilot designs for cyclic-prefix ofdm on time-varying and sparse underwater acoustic channels", in Proc. MTS/IEEE OCEANS Conf., Santander, Spain, June 2011.

[10] P. Tan and N.C. Beaulieu, "Precise ber analysis of $\pi / 4$-dqpsk ofdm with carrier frequency offset over frequency selective fast fading channels", IEEE Trans. Wireless Commun., vol. 6, no. 10, pp. 3770-3780, Oct. 2007.

[11] B.S. Sharif, J. Neasham, O.R. Hinton, and A.E. Adams, "A computationally efficient doppler compensation system for underwater acoustic communications", IEEE J. Oceanic Eng., vol. 25, no. 1, pp. 52-61, Jan. 2000.

[12] A.E. Abdelkareem, B.S. Sharif, C.C. Tsimenidis, J.A. Neasham, and O.R. Hinton, "Low-complexity doppler compensation for ofdm-based underwater acoustic communication systems", in Proc. MTS/IEEE OCEANS Conf., Santander, Spain, June 2011. 